\title{
Early tumor dissemination, but late metastasis: insights into tumor dormancy
}

\author{
Martin Röcken
}

Department of Dermatology, Eberhard Karls University, Tübingen, Germany.

\begin{abstract}
The classical model of metastasis is that tumor cell dissemination occurs late in tumor development, after the primary tumor has grown, and that only then will tumor cells invade the local tissue, enter the blood or lymphatic vessels, and colonize new sites to cause metastases. However, evidence increasingly indicates that single tumor cells spread to distant sites much earlier than previously believed. In this issue of the JCI, Eyles and colleagues provide new insight into the mechanisms underlying early tumor cell dissemination, formation of metastases, and tumor immunosurveillance using transgenic mice that spontaneously develop melanomas of the uvea. The authors provide striking evidence that tumor cells start to disseminate during the initial steps of tumor development, that late appearing metastases arise from these early disseminated tumor cells, and that $\mathrm{CD8}^{+} \mathrm{T}$ cells inhibit the growth of disseminated tumor cells, surprisingly, not by cytotoxic effects, but through cytostatic effects.
\end{abstract}

\section{Basic aspects of tumor immunosurveillance}

The original concept of tumor immunosurveillance, as stated several decades ago by F.M. Burnet and T.R. Prehn $(1,2)$, postulated that most malignant tumors that arise are eliminated via lymphocyte-mediated responses before they become clinically detectable. Having been abandoned for decades, the concept of tumor immunosurveillance has been rekindled by various observations, but its importance remains highly debated. One important contribution to the debate was the description of specific tumor-associated antigens (3), because they are a prerequisite to the argument in favor of tumor-specific immunosurveillance. Subsequently, many data have revealed that slowly developing, endogenous tumors evade the immune system by various mechanisms. For example, malignant cell clones can compromise multiple immune functions, including immune cell recognition of the tumor and immune cell-mediated killing of tumor cells (4-7). However, in early phases, tumors rarely cause harm and are often either ignored by the immune system or induce specific tolerance $(6,7)$. To date, the question of whether the immune system can specifi-

Conflict of interest: The author has declared that no conflict of interest exists.

Citation for this article: J Clin Invest. 2010; 120(6):1800-1803. doi:10.1172/JCI43424. cally recognize and control endogenously developing tumors remains widely debated (4, 7-9). In this issue of the JCI, Eyles and colleagues provide evidence in a mouse model of melanoma that tumor immunosurveillance not only controls the growth of primary malignant tumors, but also keeps metastatic outgrowth in check (10).

\section{Metastasis: a classical multistep process, or early dissemination of tumor cells?}

The risk of metastases increases with the thickness of a primary tumor. Therefore, current tumor biology concepts mainly postulate that tumor cell dissemination occurs late in tumor development and follows a classic multistep process (Figure 1A). First, the primary tumor grows; second, tumor cells invade the local tissue and enter the blood or lymphatic vessels; third, tumor cells colonize new sites to cause metastases. Yet increasing amounts of clinical data have revealed that single tumor cells spread to distant sites much earlier than previously believed. For example, single disseminated tumor cells can be found in the lymph nodes or bone marrow of healthy women with a history of early-stage breast cancer that have no clinical evidence of metastasis or tumor recurrence $(11,12)$. Women with such dormant cancer cells live with an increased risk of sudden metastases, which may occur more than a decade after primary tumor surgery. Similarly, single melanoma cells can be found in the lymph nodes of patients with thin melanomas; these single dormant tumor cells seem to be of prognostic relevance, as patients can develop metastases after more than 10 years (13). Nonetheless, more than $90 \%$ of these patients remain without metastasis for at least 10 years after surgery.

The stealth of tumor cell dissemination and the extent to which disseminated tumor cells can remain dormant is highlighted by the inadvertent transmission of melanoma from a patient who served as an organ donor following sudden brain death to the organ recipients (14). At 2 years after the donor's kidneys had been transplanted into 2 recipients, both recipients died from metastases of malignant melanoma. The donor had been treated for malignant melanoma 16 years prior to his death, but was free of secondary disease and detectable metastases at the time he died. Thus, even though free of clinical disease, the organ donor had transferred melanoma cells with the graft to the recipients, who rapidly died from metastasizing melanoma under the conditions of transplantation and transplant-associated immunosuppression.

\section{Insights into the early spread of single tumor cells from the leading edge of melanoma}

Transplanted tumor models in rodents are often used to study metastasis; however, these models often fail to recapitulate the latency between tumor cell dissemination and metastatic outgrowth in humans. Eyles et al. sought to overcome this issue - in order to determine more precisely the role of disseminated tumor cells in the growth of metastases and the mechanisms underlying dormancy - using the RET.AAD spontaneous mouse model of melanoma, in which the human RET oncogene is expressed by melanocytes, leading to their oncogenic transformation (10). They found that the first single tumor cells tended to spread very early during oncogenesis, less than 3 weeks after the clinical onset of the primary tumor. At this time, the tumors showed no histological evi- 
A

Intraepithelial neoplasia

Tumor cells have not invaded tissue Tumor cells

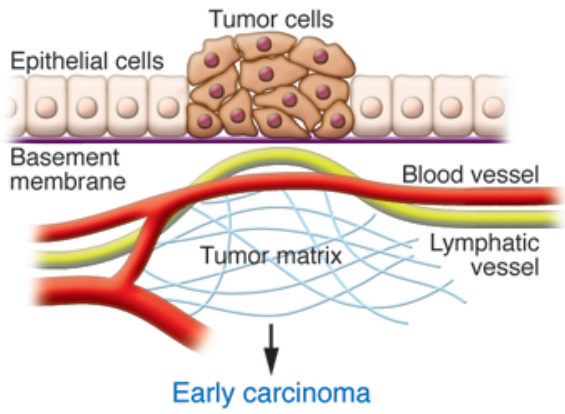

Tumor cells have invaded tissue

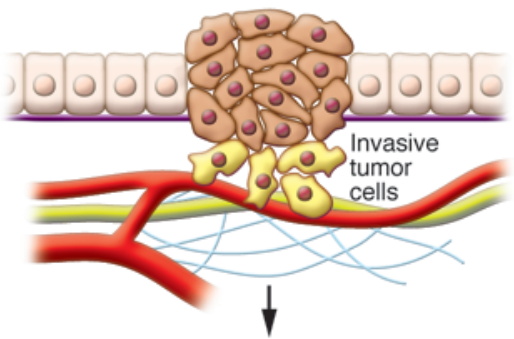

Late carcinoma

Tumor cells have entered blood and lymphatic vessels

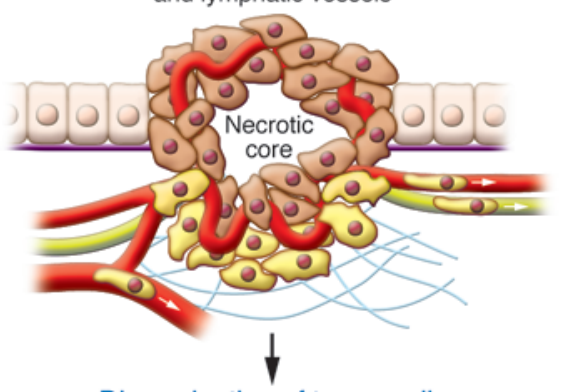

Dissemination of tumor cells in blood and lymphatic vessels

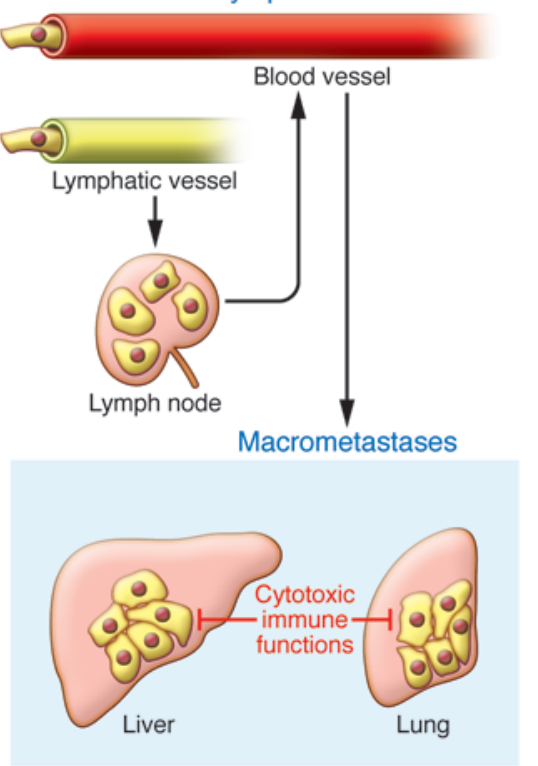

B

Intraepithelial neoplasia Tumor cells have not invaded tissue
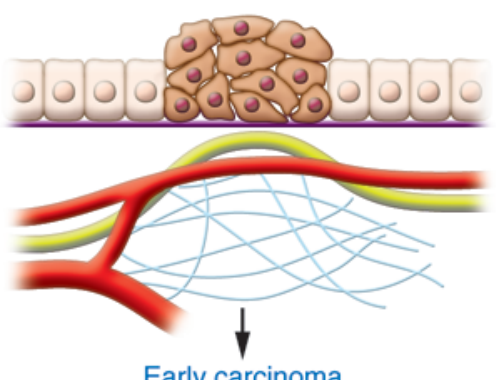

Early carcinoma

Tumor cells have invaded tissue

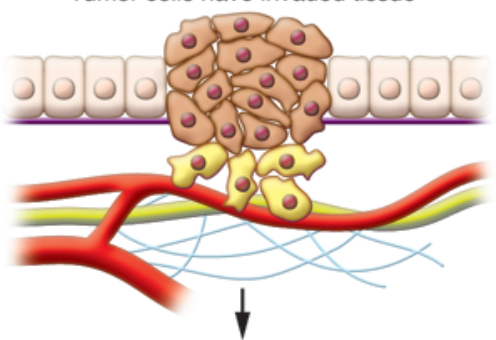

Dissemination of single tumor cells in blood and lymphatic vessels

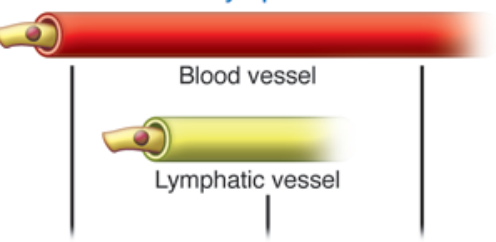

Dormancy of single tumor cells/

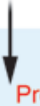
micrometastases

Prevention of macrometastasis by cytostatic and cytotoxic signals
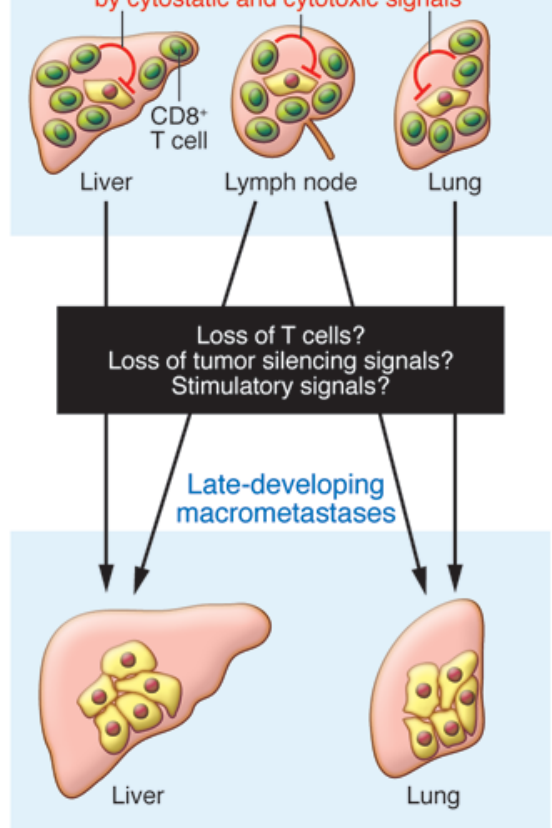

Figure 1

Models of metastasis. (A) Classical multistep model of metastasis. Here, tumor cells normally arise from late, vessel-infiltrating cancers. It is believed that spreading tumor cells either fail to enter target organs or are controlled by cytotoxic effects (lysis or apoptosis). (B) Model of metastasis as suggested by Eyles et al. (10). Single tumor cells start to spread much earlier than expected and form single-cell metastases. Such single-cell metastases remain dormant for long periods of time, also through cytostatic signals that reduce tumor cell proliferation. In the absence of such cytostatic signals, metastases start to grow rapidly.

dence for invasion of small vessels. Detectable metastases became apparent much later in life, up to 1.5 years, which suggests that disseminated tumor cells remained dormant for long periods during the life of the mice (10). To determine whether the metastases were really derived from the early spreading tumor cells, or whether they followed a classical multistep progression, the authors compared the genomes of primary tumors with those of late metastases. As the late metastases bore a genetic profile similar to that of the primary tumor, and profiles were specific for each individual mouse, it seems that the metastases arose from the early disseminated tumor cells (10). Thus, both the early spread of single tumor cells and the latency between tumor cell dissemination and metastatic outgrowth observed in humans were modeled in RET.AAD mice (Figure 1B).

Early tumor cell dissemination reminiscent of the events in human metastasis has also been analyzed by Husemann et al. (15) in 2 distinct transgenic mouse strains that model breast cancer. Although these authors did not study the latency between tumor cell dissemination and metastatic outgrowth, they did show that, upon adoptive transfer, disseminated tumor cells are capable of homing to the bone marrow and causing metastases. It is interesting to note that in these breast cancer models, the tumor cells tended to spread to the bone marrow and the lung, whereas in the spontaneous melanoma model used by Eyles et al., cells spread more diffusely (10). Importantly, in both studies, apparent metastatic cells bore the genetic signature of early tumor development $(10,15)$, further underlining that tumor cell dissemination is an early event. The data discussed here indicate that tumor cells disseminate to 
distant sites early during primary tumor development, but do not cause metastasis (Figure 1B). Understanding why these disseminated tumor cells fail to cause early metastasis and what events are responsible for tumor dormancy are key to determining whether new therapeutic interventions can be developed for the human situation.

\section{Evidence for immune-mediated tumor dormancy in the absence of tumor cell killing}

To uncover potential mechanisms of tumor dormancy, Eyles et al. depleted $\mathrm{CD}^{+} \mathrm{T}$ cells in RET.AAD mice (10). This T cell depletion markedly increased the risk of overt metastases, directly showing a critical role for $\mathrm{T}$ cell-dependent tumor immunosurveillance in the early metastatic stage of this melanoma model. The current view of $\mathrm{T}$ cell-mediated tumor immunosurveillance is that $\mathrm{CD}^{+} \mathrm{T}$ cells control tumor cells through classic death-inducing mechanisms $(5,9,10)$. Although $\mathrm{CD}^{+} \mathrm{T}$ cell depletion was the method used by Eyles et al. to uncover a role for $\mathrm{T}$ cell-dependent tumor immunosurveillance in keeping disseminated tumor cells from forming metastases, the authors present an additional, surprising piece of data contradicting this classic hypothesis: In the presence of $\mathrm{CD}^{+} \mathrm{T}$ cells, the number of $\mathrm{Ki}^{+} 7^{+}$tumor cells is low, whereas the number of $\mathrm{Ki} 7^{+}$ tumor cells is high in the absence of $\mathrm{CD}^{+}$ $\mathrm{T}$ cells. As expression of $\mathrm{Ki} 67$ is associated with cell proliferation and cell cycle progression, these data strongly argue that, besides the known cytotoxic effects, a cytostatic mode of tumor silencing may be keeping the disseminated tumor cells from forming metastases (Figure 1B).

Müller-Hermelink et al. recently showed that immune-mediated arrest of endogenous tumor development is associated with a substantial reduction in tumor cell proliferation (16). Using BrdU incorporation, apoptosis assays, and $\mathrm{CD}^{+} \mathrm{T}$ cell depletion, they found that $\mathrm{CD} 4^{+} \mathrm{Th} 1$ cells could induce a state of tumor dormancy by reducing tumor cell proliferation and cell cycle progression through strictly IFN- $\gamma-$ and TNF-dependent signals. Arresting tumor growth required neither detectable signs of tumor cell killing nor apoptosis. This is in line with other recent reports showing that $\mathrm{T}$ cell immunity can induce tumor dormancy with little or no signs of either tumor cell killing or apoptosis (17-19). In all of these reports, tumor dormancy required IFN- $\gamma$ - or TNF-mediated signal- ing. Interestingly, this is in accordance with an increasing number of studies in humans showing that immunotherapy with type I IFNs or vaccine strategies that induce type II IFN-producing Th1 immune responses are needed to inhibit tumor progression and prolong overall survival $(7,9,20,21)$. The importance of both type I and type II IFNs has been extensively studied in experimental mice (9) and is underlined by surprising data from humans as well as from experimental animals: in the absence of IFNs or Th1 cells, tumor immunity not only fails to protect against tumors, but can even promote tumor progression (16, 19, 20). That said, it will be important to uncover the mechanisms underlying tumor dormancy elicited by type I or type II IFNs; it has been proposed that antiangiogenic effects might contribute, as some tumor cells may not express appropriate IFN receptors. Yet, in a number of clinical trials, antiangiogenic therapies were less efficient than hoped, and some tumors may even develop a more aggressive phenotype in the presence of antiangiogenic drugs (22). It is therefore unlikely that the tumor-protective effects of IFNs can be reduced to their antiangiogenic effects.

\section{Can inhibition of cell cycle progression cause tumor dormancy?} Today it is known that disseminated single tumor cells rarely cause metastases, and if they do, it is frequently many years after they reached their distant site. However, the mechanisms underlying tumor dormancy remain a major mystery. MüllerHermelink et al. (16) and Eyles et al. (10) independently found that tumor immunosurveillance correlated with a substantial reduction in either $\mathrm{BrdU}^{+}$or $\mathrm{Ki} 7^{+}$tumor cells, key findings characteristic of inhibition of cell proliferation and cell cycle progression, and that tumor-specific $T$ cells prevented tumor growth in the absence of histological evidence for major tumor cell killing or apoptosis. It is therefore difficult to imagine that the immune system controls disseminated tumor cells only by mechanisms of direct killing; rather, it is likely that immunosurveillance also induces tumor dormancy through other mechanisms that are still poorly investigated. IFNs and TNF both are not only involved in killing and apoptosis; they have pleiotropic effects on the metabolism of somatic cells through the control of signaling cascades that regulate cell proliferation and cell cycle progression and the control of signaling molecules that influence cell differentiation and the process of transformation $(9,10,16)$. Therefore, immunosurveillance may indeed induce dormancy in single, disseminated tumor cells by arresting their cell cycle through cytokine-mediated signals. This would also explain why late metastases might still bear the genetic signature of early disseminated tumor cells $(10,15)$.

\section{Perspectives}

These insights, arising from a number of observations in mice and humans, reveal that the classical immunological concept of tumor eradication by tumor cell killing should be abandoned. They raise important questions that should affect the design of future immune therapies. If single tumor cells emigrate early, why do overt metastases appear only at later tumor stages? Is this a question of quantity, or are there specific signals? How does the immune system control single, disseminated tumor cells and micrometastasis and establish tumor dormancy, and what awakens disseminated tumor cells and makes them grow into metastases?

\section{Acknowledgments}

I thank H. Braumüller, T. Wieder, K. Ghoreschi, and G. Riethmüller for helpful discussions and critically reading the manuscript. The author was supported by SFB 685, Wilhelm Sander Foundation (2005.043.2), and Deutsche Krebshilfe (no. 109037).

Address correspondence to: Martin Röcken, Department of Dermatology, Eberhard Karls University, Liebermeisterstr. 25, 72076 Tübingen, Germany. Phone: 49.7071.2984556; Fax: 49.7071.295450; E-mail:mrocken@med.uni-tuebingen.de. lance. Prog Exp Tumor Res. 1970;13:1-27.

2. Prehn RT. Immunosurveillance, regeneration and oncogenesis. Prog Exp Tumor Res. 1971;14:1-24.

3. Knuth A, Wolfel T, Klehmann E, Boon T, Meyer zum Buschenfelde KH. Cytolytic T-cell clones against an autologous human melanoma: specificity study and definition of three antigens by immunoselection. Proc Natl Acad Sci U S A. 1989; 86(8):2804-2808

4. Qin Z, Blankenstein T. A cancer immunosurveillance controversy. Nat Immunol. 2004;5(1):3-4.

5 . Mocikat R, et al. Natural killer cells activated by MHC class I(low) targets prime dendritic cells to induce protective CD8 T cell responses. Immunity. 2003;19(4):561-569.

6. Willimsky G, Blankenstein T. Sporadic immunogenic tumours avoid destruction by inducing T-cell tolerance. Nature. 2005;437(7055):141-146

7. Finn OJ. Cancer immunology. N Engl J Med. 2008; 358(25):2704-2715 
8. Shankaran V, et al. IFNgamma and lymphocytes prevent primary tumour development and shape tumour immunogenicity. Nature. 2001; 410(6832):1107-1111.

9. Dunn GP, Koebel CM, Schreiber RD. Interferons, immunity and cancer immunoediting. Nat Rev Immunol. 2006;6(11):836-848.

10. Eyles J, et al. Tumor cells disseminate early, but immunosurveillance limits metastatic outgrowth, in a mouse model of melanoma. J Clin Invest. 2010;120(6):2030-2039.

11. Pantel K, Alix-Panabières C, Riethdorf S. Cancer micrometastases. Nat Rev Clin Oncol. 2009; 6(6):339-351.

12. Riethmuller G, Klein CA. Early cancer cell dissemination and late metastatic relapse: clinical reflections and biological approaches to the dormancy problem in patients. Semin Cancer Biol. 2001; 11(4):307-311.
13. Ulmer A, et al. Detection of melanoma cells displaying multiple genomic changes in histopathologically negative sentinel lymph nodes. Clin Cancer Res. 2005;11(15):5425-5432.

14. MacKie RM, Reid R, Junor B. Fatal melanoma transferred in a donated kidney 16 years after melanoma surgery. N Engl J Med. 2003;348(6):567-568.

15. Husemann Y, et al. Systemic spread is an early step in breast cancer. Cancer Cell. 2008;13(1):58-68.

16. Muller-Hermelink N, et al. TNFR1 signaling and IFN-gamma signaling determine whether T cells induce tumor dormancy or promote multistage carcinogenesis. Cancer Cell. 2008;13(6):507-518.

17. Koebel CM, et al. Adaptive immunity maintains occult cancer in an equilibrium state. Nature. 2007;450(7171):903-907

18. Zhang B, Karrison T, Rowley DA, Schreiber H. IFNgamma- and TNF-dependent bystander eradication of antigen-loss variants in established mouse cancers. J Clin Invest. 2008;118(4):1398-1404

19. Ziegler A, et al. EpCAM, a human tumor-associated antigen promotes Th2 development and tumor immune evasion. Blood. 2009;113(15):3494-3502.

20. Schadendorf D, et al. Dacarbazine (DTIC) versus vaccination with autologous peptide-pulsed dendritic cells (DC) in first-line treatment of patients with metastatic melanoma: a randomized phase III trial of the DC study group of the DeCOG. Ann Oncol. 2006;17(4):563-570.

21. Hauschild A, et al. Efficacy of low-dose interferon \{alpha\}2a 18 versus 60 months of treatment in patients with primary melanoma of $>=1.5 \mathrm{~mm}$ tumor thickness: results of a randomized phase III DeCOG trial. J Clin Oncol. 2010;28(5):841-846.

22. Loges S, Mazzone M, Hohensinner P, Carmeliet P. Silencing or fueling metastasis with VEGF inhibitors: antiangiogenesis revisited. Cancer Cell. 2009;15(3):167-170.

\title{
Gazing into a crystal ball to predict kidney transplant outcome
}

\author{
Bernd Schröppel ${ }^{1}$ and Peter S. Heeger ${ }^{2}$
}

${ }^{1}$ Mount Sinai School of Medicine, New York, New York, USA. ${ }^{2}$ Recanati Miller Transplantation Institute, New York, New York, USA.

\begin{abstract}
Kidney transplantation is the optimal therapy for end-stage kidney disease but requires lifelong immunosuppression. Despite improvements in immunosuppression regimens that have reduced rates of acute transplant rejection, long-term allograft survival remains suboptimal. More than $50 \%$ of transplanted kidneys from deceased donors fail within 10 years. In order to improve long-term outcomes, physicians need to better understand mechanisms underlying transplant rejection and tolerance in humans. They also need biomarkers that differentiate patients likely to maintain excellent and stable allograft function from recipients at risk of losing their transplants. By studying kidney transplant recipients at high risk for graft loss and rare, spontaneously tolerant kidney transplant recipients, researchers reporting in 3 papers in this issue of the JCI shed new light on these topics.
\end{abstract}

\section{Late graft failure after kidney transplantation}

Kidney transplantation is the most common solid organ transplant procedure carried out in the US; more than 16,000 such transplants were performed in 2009 (1). Advances in immunosuppression over the past 2 decades have drastically reduced the incidence of acute $\mathrm{T}$ cell-mediated rejection episodes, but have not significantly improved long-term allograft survival (2). The actual half-life for a transplanted kidney from a deceased donor (the most common source of kidneys for transplantation) is only 8 years (2), and as few as

Conflict of interest: The authors have declared that no conflict of interest exists.

Citation for this article: J Clin Invest. 2010; 120(6):1803-1806. doi:10.1172/JCI43286. one-third of kidney allografts obtained from older donors remain functioning 10 years later (3). Allograft dysfunction can result in the need for a further transplant and is a strong independent predictor of recipient cardiovascular mortality (4). Late graft loss is commonly caused by chronic allograft nephropathy, characterized by tissue fibrosis and tubular atrophy (5). Among the factors known to contribute to its pathogenesis are uncontrolled alloimmune reactivity ( $T$ cell- and/or antibody-mediated injury), recurrent primary renal disease, hypertension, diabetes, and drug toxicity. With regard to the latter, kidney toxicity as a result of long-term treatment with the immunosuppressive agents tacrolimus or cyclosporine is detectable in virtually all transplanted kidneys (6).
Although research progress has improved our knowledge of the prevalence, clinical significance, and pathogenesis of chronic kidney allograft injury, research has not yet influenced therapeutic decision making to improve outcomes. Currently, posttransplant care, including immunosuppression, is protocol driven. Alterations in drug dosing are made based on center-derived protocols and physician experience. Because chronic injury is multifactorial, a one-size-fits-all approach is not ideal. To develop individual therapeutic approaches tailored to individual patients, transplant physicians need risk assessment tools that can discriminate specific pathogenic mechanisms, prospectively identify those transplant recipients destined to have excellent graft function, recognize those recipients at high risk for graft loss, and ultimately guide specific changes in therapy. In this issue of the JCI, 3 papers provide new data that move us closer to these goals (7-9).

\section{An intragraft molecular signature can predict graft loss}

To assess the risk of future graft loss, Einecke and colleagues (7) evaluated gene expression profiles in tissue from 105 kidney graft biopsies that were performed to determine the cause of allograft dysfunction and/or proteinuria using microarray 